\title{
Ueber angebliche Blaublindheit der Fovea centralis.
}

Von

\section{Ewald Hering,}

Professor an der deutschen Universität Prag.

Im dritten Abschnitte einer Abhandlung „uber den menschlichen Sehpurpur und seine Bedeutung für das Sehen "1) behauptet Arthur König, "dass die Fovea blaublind ist". „Es haben also", wie er sagt, „trichromatische Personen hier ein dichromatisches, und dichromatische ein monochromatisches Farbensystem ${ }^{2}$ )." Die Grösse des blaublinden Bezirkes entspricht nach $\mathrm{K} \ddot{o} \mathrm{n} \mathrm{i} g$ einem Gesichtswinkel von 55 bis 70 Winkelminuten. Auf einem $30 \mathrm{~cm}$ vom Knotenpunkte des Auges entfernten Gesichtsfelde müsste hiernach in einem Felde von 5 bis $6 \mathrm{~mm}$ Durchmesser, dessen Mittelpunkt von der Gesichtslinie getroffen wird, alles Blaue als solehes unkenntlich sein.

Da das tägliche Leben Jedem, der darnach sucht, Gelegenheit giebt, durch Betrachtnng hinreichend kleiner farbiger Objecte den Farbensinn seiner Fovea zu prüfen and sich von deren vortrefflichem Blausinn zu überzeugen, so läge für mich keine Veranlassung vor, die offenbar irrige Behauptung $\mathrm{K} \ddot{\mathrm{n}} \mathrm{n} \mathrm{g}$ 's hier zu besprechen, wenn ich nicht dabei Gelegenheit bätte, die Unzuverlässigkeit der $\mathrm{K}$ ö $\mathbf{n}$ ig'schen Farbengleichungen an einem relativ einfachen und zugleich schlagenden Beispiele darzulegen. Die meisten physiologischen Speculationen König's aber gründen sich auf seine Farbengleichungen.

Dass die Fovea blaublind sei, sucht $\mathrm{K} \ddot{\mathrm{n}} \mathrm{ig}$ zuerst damit zu beweisen, „dass an einer Reihe von monochromatisch leuchten-

1) Sitzungsber. d. Berliner Akad. vom 21. Juni 1894.

2) Unter trichromatischen Personen versteht $\mathrm{K}$ ö nig die Farbentüchtigen, unter dichromatischen die partiell, und unter monochromatischen die total Farbenblinden. 
den blauen Punkten, deren Bild man quer durch die Fovea legt, bei geeigneter Intensität die auf die Fovea fallenden verschwinden", und dass es ihm ,nach längerer Uebung gelingt, den Mond in der Fovea verschwinden zu lassen“, wenn er "ein nu r blaue Strahlen durchlassendes Glas" ${ }^{1}$ ) vor sein Auge hält.

Richtet man das einige Zeit geschlossen oder ganz verdunkelt gewesene Auge auf eine von schwachem blauen Lichte beleuchtete Fläehe, so sieht man bekanntlich den Maxwell'schen Fleck besonders deutlich u. zw. je nach den Umständen dunkelblau oder anch fast schwarz. Donders drüekte dies dadurch aus, dass er sagte, man habe unter solchen Umständen ein centrales, ,Skotom". Unter den von $\mathrm{K} 00 \mathrm{n} \mathbf{i g}$ benützten Versuchsbedingungen kommen die erwähnten, objectiv nur schwach leuchtenden oder durch das blaue Glas sebeinenden Objecte in das Bereich des Maxwell'schen Fleckes bezw, auf die Stelle des directen Sehens zu liegen und werden hier unter sonst gtustigen Umständen unsichtbar. Können doch nach der alten und oft nachuntersuchten Erfahrung der Astronomen auch schwach weissleuchtende Objecte, wie z. B. Sterne, bei direkter Betrachtung versehwinden, während sie indirekt ganz gut gesehen werden. Da man jedoch unter den Kö n ig'schen Fersuchsbedingungen die blau leuchtenden Objecte, wenn man ihre Lichtstärke zureichend vergrössert, beim Fixiren besonders schön blau sieht, nämlich gesättigter blau als bei indirekter Betrachtung, so sind solche Versuche zwar recht geeignet, den vorzuiglichen Blausinn der centralen Netzhaut und insbesondere auch der Fovea zu beweisen, nicht aber das Gegentheil. Deshalb erscheint es auch nicht nöthig, das interessante und bereits wiederholt untersuchte Verhalten der mittlen Netzhaut und ihrer Fovea gegen-

1) Ein solches Glas, dass nur blaue Strahlen durchlässt, besitze ich noch nicht. Die blauen Kobaltgläser, die ich und viele Andere zu benutzen pflegen, habon bekanntlich ein verwickeltes Absorptionsspectrum. Luegt man eine genügende Zahl solcher Gläser übereinander, so lassen sie vom Tageslichte nur noch den röthlichblauen (indigoblauen) und violetten Theil des Spectrums mit merklicher Helligkeit durch, vom kïnstlichen Lichte (Gaslicht, Petroleumlicht, Auer-Glühlicht) aber überdies noch viel Roth. Hält man eine genügende Anzahl soleher Gläser vor das Auge, so kann der Mond für die centrale Netzhaut unkenntlich werden, wobei natürlich viel auf den jeweiligen Ermüdungszustand derselben mit ankommt. Bei Benutzung grüner Gläser ist der Versuch schon etwas sehwieriger. 
über schwach blau oder sonstwie leuchtenden kleinen Objecten bei hell- und bei dunkeladaptirtem Auge in Dunkelraume hier weiter zu besprechen.

Diese Versuche König's bieten also, abgesehen von der irrigen Schlussfolgerung, nichts wesentlich Neues. Völlig neue Thatsachen jedoch wïrden sich aus seinen Farbengleichungen ergeben, wenn sie richtig wären. Könnte der Leser nach der Beschreibung, welche König von dem „Verschwinden" des durch ein blaues Glas fixirten Mondes giebt, auf die Vermuthung kommen, dass König sich die Fovea als völlig unerregbar durch blaue Strahlen denke, so lehren diese Gleichungen, dass dies nicht seine Meinung ist, sondern dass er der Fovea nur das Vermögen, die blane „Grundempfindung" zu, erzengen, absprechen will, im Uebrigen aber der Ansicht ist, dass blaues Licht die rothe und die grine „Grundempfindung“ auch auf der Fovea za erwecken vermöge; ein bestimmtes Roth, Grün und Blau sind nämlich nach seiner Ansicht die drei Grundempfindungen. Hiernach also wäre die Fovea "dichromatisch".

Ich habe vor einiger Zeit auf die Eigenthümlichkeit der Farbengleichungen hingewiesen ${ }^{1}$ ), welche in einem viel kleineren Felde hergestellt werden, als sonst gebräuchlich ist. Kön i $\mathrm{g}$ versuchte nun, spectrale Farbengleichungen in so kleinem Felde herzustellen, dass das Bild desselben auf der Fovea Platz hatte. Die eine Hälfte des Feldes wurde mit je einem passenden Gemisch aus rothem Lichte von $650 \mu \mu$ und blanem Lichte von $475 \mu \mu$ beleuchtet, die andere mit je einem im Spectram zwisehen den beiden eben genannten liegenden einfachen Liche. Er behauptet, dass sich $z u$ jedem dieser einfachen Lichter ein passendes Gemisch aus den beiden erstgenannten ( 650 und $475 \mu \mu$ ) herstellen liess, welches bei entsprechender Intensität dem einfachen Lichte ganz gleich schien.

1) Ueber den Einfluss der Macula lutea auf spectrale Farbengleichungen. Dieses Arch. Bd. 54. S. 277. 1893. In dieser Abhandlung erwähnte ich die Angabe Max Schultze's über die gelbe Pigmentirung der Fovea centralis, welche in Widerspruch steht mit den Angaben andrer Autoren. Dnrch die Güte meines Collegen Gussenbauer hatte ich bald nachher Gelegenheit, ein ganz frisches Auge zu untersuchen, in welchem sich die Retina ohne den als Foramen centrale bekannten Defect isoliren liess. Mir schien ebenfalls die Mitte der Macula pigmentfrei, die Zone stärkster gelber Pigmentirung aber der Mitte am nächsten.

E. Pflüger, Archiv f. Phygiologie. Bd. 69. 
„Die völlige Dichromasic meiner Fovea", so sagt er (S. 592 [16]), „habe ich nachgewiesen, indem ich innerlalb derselben Farbengleichungen zwischen Mischungen von $650 \mu \mu$ und $475 \mu \mu$ einerseits und allen dazwischen liegenden Spectralregionen anderseits herstellte."

Man ist zunächst iuberraseht, bei allen diesen Gleichungen das Licht $\operatorname{von}^{-4} 45 \mu \mu$ verwendet zu finden; denn dieses Licht ist blau, und blaues Licht sollte nach König's eben besprochenen Angaben die Fovea so wenig erregen, dass es sehr leicht „, verschwindet." Diesem Uebelstande musste also dadurch abgeholfen werden, dass das blaue Licht von $475 \mu \mu$ bei grösserer Intensität angewendet wurde, 'als das blaue Licht der auf der Fovea verschwindenden Lichtpunkte oder des durch das blaue Glas gesehenen Mondes. Man vermisst jedoch eine Mittheilung darüber, wie für König das zu allen seinen Gleichungen verwendete blaue Licht ausgesehen hat, wenn er damit seine Fovea bei jener Intensität beleuchtete, bei der es auf derselben nicht mehr versehwand, und ob es da wirklich gelbgriun aussah, wie dies nach seinen "Grundempfindungscurven" (s. u.) der Fall sein mitsste, wenn seine Fovea wirklich „blaublind“" wäre. Mir erscheint dieses Licht, mit der Fovea bei passender Intensität gesehen, selbstverständlich schön blau, und andern "Trichromaten" auch.

Die Farbentöne, welche durch Mischung des genannten Blan von $475 \mu \mu$ und Roth von $650 \mu \mu$ erzeugt werden können, sind Indigoblau, Violett, Violett-Purpur, Roth-Purpur, kurz alle jene Töne, welche im Farbenzirkel die Uebergänge vom Blau dureb Purpurroth hindurch bis zum gelblichen Roth des Spectrums bilden. Dies sind also die Farben, welche auf der einen Seite der erwähnten Gleichungen erscheinen konnten. Die Farbentöne aber, welche den im Spectrum zwischen Roth von $650 \mu \mu$ und Blan von $475 \mu \mu$ liegenden einfachen Lichtern entsprechen, nämlich Orange, Gelb, Grïngelb, Grün, Grünblau und deren Zwischentöne sind jene Farben, welche auf der andern Seite der Gleichungen vorkommen konnten. Man erkennt sofort, dass derartige Gleichungen nur bei Roth-Grünblindheit ( Rothblindheit" oder "Grünblindheit") möglich sein würden. Denn für den Roth-Grünblinden ist das Licht von $650 \mu \mu$ ein Gelb von grösserer Sättigung als alle übrigen Lichter kleinerer Wellenlänge bis zu der sogenannten neutralen Stelle seines Spectrums, und das Licht von $475 \mu \mu$ ist für ihn ein Blau von grösserer Sättigung als alle Lichter grösserer Wellen- 
länge bis zu dieser neutralen Stelle: folglich kann für ihn aus einem passenden Gemisch dieser beiden Lichter eine Gleichung mit jedem zwischen $650 \mu u$ und $475 \mu \mu$ gelegenen einfachen Lichte hergestellt werden.

Für König haben wahrscheinlich derartige Ueberlegungen kein Gewicht, und ich könnte auch nicht hoffen, ihn durch dieselben von seinem Irrthum zu überzeugen. Denn er betrachtet die Erscheinungen des Farbensinns vom Standpunkte seiner drei "Grundempfindungscurven" ${ }^{1}$ ), ,obwohl denselben, seit auch Helmholtz sie nicht gelten liess, ausser von König und seinen Mitarbeitern kaum noch von irgend Jemand eine reale Bedeutung beigelegt wird. Diese "Grundempfindungscurven" hält König, wie er (S. 596 [20]) ausdrücklich bemerkt, auch jetzt noch für richtig, sofern man nur die bezüglichen Gleichungen bei hinreichender Lichtintensität herstelle; für "niedrigere Helligkeiten "sseien diese Curven allerdings nicht mehr "berechtigt", seit es sich herausgestellt babe, dass für solche das Newton'sche Mischungsgesetz nicht guiltig sei. Solche niedrige Intensitäten waren, wie aus dem oben Gesagten folgt, bei Herstellung der Gleichungen für die Fovea ausgeschlossen, weil andernfalls das blaue Licht von $475 \mu \mu$, welches stets mit benützt wurde, unter dem Schwellenwerthe geblieben und „verschwunden" wäre.

Nun sind aber, wenn man sich einmal auf den König'schen Standpunkt stellen und seine „Grundempfindungscurven" gelten lassen will, die von ihm für die Fovea bebaupteten Farbengleichungen ebenso unmöglich, wie sie anch in Wirklichkeit für den von $\nabla$ on Vin t s e h g alu und mir untersuchten Blaugelbblinden unmöglich waren. Dies ist so offenbar, dass ich mich der Hoffnung hingebe, auch König selbst davon zu überzeugen.

Nach den König'schen Carven für die rothe und grüne

1) Die Grundempfindungen und ihre Intensitätsvertheilung im Spectrum. Sitzungsber. der Berliner Akad, vom 29. Juli 1886. Dass es ganz unzulässig ist, Curven, welche die Vertheilung angenommener Componenten des optischen Reizwerthes der Lichter im Spectrum darstellen sollen, als Empfindungscurven zu bezeichnen, habe ich schon einmal betont. Die strenge begriffliche Unterscheidung des optischen Reizwerthes eines Lichtes von der Empfindung, die es uns erweckt, ist eine der unentbehrlichsten Voraussetzungen einer klaren Behandlung der Farbenlehre. 
Grundempfindung bezw. nach der entsprechenden Tabelle (l; c. S. 827 [23]) hat, bezogen auf einen bestimmten Einheitswerth, Licht von $650 \mu \mu$ den Rothwerth 1,70 und den Grïnwerth 0,38 ; " $475 \mu \mu$ " $\quad 0,83$ " $\quad$ " 1,36 . Im Lichte von $475 \mu \mu$ ist also das Verhältniss des Grïnwerthes zum Rothwerthe, d. i. $\frac{G}{R}=1,64$. In allen (für uns vorwiegend grïnen) Lichtern zwischen den Fra u en h ofe r'sehen Linien $E$ und $F$ ist aber nach Kön ig's eigenen Tabellen und Curven dieses Verhältniss ein wesentlich grösseres, z. B. für $516,5 \mu \mu=2,04$; für $505 \mu \mu=2,26$; für $495 \mu \mu=2,28$. Folglich wäre es für Blaublinde unmöglich, aus einem Gemisch von $650 \mu \mu$ und $475 \mu \mu$ eine Gleichung mit den zwischen den Linien $E$ und $F$ des Spectrums gelegenen Lichtern herzustellen. Denn wenn man dem Lichte von $475 \mu \mu$, in welchem das Verhältniss $\frac{G}{R}$ bereits nur 1,64 beträgt, das Licht von $650 \mu \mu$ beimiseht, dessen Rothwerth über viermal grösser sein soll, als sein Grünwerth, so wird selbstverständlich in dem Gemisch der Grïnwerth im Verhältniss zum Rothwerth stets kleiner, nie aber grösser als 1,64, wie dies doch zur Herstellung einer Gleichung mit.den einfachen Lichtern zwischen den Linien $E$ und $F$. nöthig wäre ${ }^{i}$.

Wenn also König bebauptet, e r habe, ,innerhalb der Fovea Farbengleichungen zwischen Mischungen $v 0 \mathrm{n} 650 \mu \mu$ u. $475 \mu \mu$ einerseits und allen dazwischen liegenden Spectralregionen anderseits" hergestellt, sobehauptet er etwas nach seinen eigenen Curven und Tabellen (über die Vertheilung der Roth-Grünwerthe im Speetrum seines eigenen Auges) bei Blaublindheit Unmögliches; ஜganz abgesehen davon, dass es $\mathfrak{u}$ berbaupt $n$ icht richtig ist."

1) Ein kritischer Blick auf sein eignes Farbendreieck, wie er es auf Grund der erwähnten Tabellen construirt und u. A. in No. 50 der "Daturwissenschaftlichen Rundschan" (Extrabeilage) abgebildet hat, . hätte K ön ig über den Widerspruch belehren können, in den er durch die angeführten angeblichen Gleichungen mit sich selbst gekommen ist. Denkt man sich aus dem mit "Blau" bezeichneten Eckpunkte dieses Dreiecks eine Gerade durch den, dem Lichte $475 \mu \mu$ entsprechenden Punkt der Curve der homogenen 
Fïr einen "Rothblinden" oder "Grünblinden" (d. b. für einen Roth-Grïnblinden) kann man allerdings anch nach den Kö ni g'schen Curven zwischen je einem Gemisch aus den beiden von ihm benutzten Lichtern und jedem im Spectrum zwischenliegenden homogenen Lichte Gleichungen herstellen. Unbedenklich hat nun König dasselbe für die "Blaublindheit" angenommen, für welche es keinen Sinn hat; und doch behauptet er, schon Fälle von Blaublindheit untersucht und diagnosticirt zu haben.

Es ist ausgeschlossen, dass die Zahl 475 ein Druckfehler sein kann, denn nehmen wir ein Licht kleinerer Wellenlänge, so wird der Widerspruch anf Grund der König'schen Curven nur noch auffallender, und ein Licht wesentlich grösserer Wellenlänge (z. B. $485 \mu \mu)$ hätte auch keinen Sinn.

Der höchst sonderbare Sachverhalt ist also folgender: König schliesstaus Versuchen, mit denen sich bei einiger Abänderung leichit der vortreffliche Blausinn der Fovea erweisen lässt, dass letztere blaublind sei. Diese Blaublindheit beweist er dann mit Hülfe von Farbengleichungen, welche, wenn sie richtig wären, z war Roth-Grünblindhe it ("Rothblindheit" oder "Grünblindbeit"), nicht aber "Blaublindheit" der Fovea beweisen könnten. In Wirklichkeit aber ist die Fovea weder „blaublind" noch roth-grünblind, sondern eminent farbentü $\mathrm{c} b \mathrm{t}$ i g.

Bei solchen unglaublich scheinenden Widersprïchen könnte jemand auf den Gedanken kommen, dass in einem Apparate für spectrale Farbenmischung das Erkennen der Farbe auf einem so kleinen Felde, wie König es benutzte, ganz unsicher sei. Dies

Lichter bis zur gegenüberliegenden Dreieckseite gezogen, so schneidet diese Gerade die Curve nochmals in einem Punkte, dessen zugehöriges homogenes (grüngelbes) Licht zwischen Gelb von $575 \mu \mu$ und Grün von $526 \mu \mu$ liegt. Nur diejenigen Lichter, welche rothwärts von jener Geraden zwischen diesem grüngelben und dem rothen lichte von $650 \mu \mu$ gelegen sind, lassen eine Gleichung mit einem Gemisch aus $650 \mu \mu$ und $475 \mu \mu$ zu. Alle homogenen Lichter aber, welche auf der Curve grünwärts von der Geraden zwischen jenem grüngelben und dem blauen Lichte von $475 \mu \mu$ liegen, lassen ${ }_{x-1}$ eine solche Gleichung nicht za. Sie nehmen eine beiläufig doppelt so lange Strecke auf der Curve ein, als die zu den genannten Gleichungen verwendbaren Lichter. 
ist aber für ein normales Auge nicht der Fall, wenn man nur nicbt unter Bedingungen arbeitet, welche überhaupt die Farbenwabrnehmung beeinträchtigen, aiso z. B. zu starke Ermüdung der centralen Netzhaut, zu weit vorgeschrittene Dunkeladaptation des Auges bei allzu geringer Intensität des benützten Lichtes. Ich babe genïgend viel mit spectralen Lichtern in sehr kleinem Felde zu thun gehabt, um dies mit voller Bestimmtheit aussprechen zu können. Man kann die entsprechend kleine Oeffnnng eines Diaphragmas nach einander mit allen Lichtern des Spectrums erleuchten und wird beim Fixiren der Mitte des Feldes die einzelnen Farben ganz deutlich unterscheiden ${ }^{1}$ ), wenn man ihnen nur eine irgend passende Helligkeit gibt, und die centrale Netzhaut sich nicht in einem extremen Zustande befindet. Ebenso kann man in solch kleinem Felde die durch Mischung von Roth $(650 \mu \mu)$ und Blau $(475 \mu \mu)$ herstellbaren Farbentöne sichtbar machen und sich überzeugen, wie gut man Violett, bläulichen Purpur und Rothpurpur bei direkter Betrachtung unterscheidet, wenngleich die Deckung der beiden gemischten Lichter wegen ihrer so versehiedenen Brechbarkeit keine genaue ist. Farbengleichungen werden selbstverständlich in so kleinem Felde stets viel zu wünschen uibrig lassen.

Leider theilt $\mathrm{König} \mathrm{gar} \mathrm{nicht} \mathrm{mit,} \mathrm{in} \mathrm{welcher} \mathrm{Farbe} \mathrm{er} \mathrm{mit}$ seiner Fovea beide Hälften des kleinen Feldes gesehen hat, während eben eine der vermeintlichen Gleichungen auf demselben eingestellt war. Nur iiber das Gelb von $580 \mu \mu$ sagt er, dass dasselbe gleich dem unzerlegten Sonnenlichte erschienen sei, sagt aber nicht, ob ihm beide gelb, wie es seine "Grundempfindungscurven" fordern, erschienen seien, oder etwa beide weiss. Welche Farbe er gesehen hat, wenn z. B. ein für uns gritines einfaches Licht einem bestimmten Gemische aus dem erwähnten rothen und blauen Lichte gleich ersehien, darüber sagt er nichts, 0 obwohl dies doch von be-

1) Hat man sich längere Zeit in einem künstlich Jerleuchteten Raume aufgehalten, und hat sich dabei das Ange für die gelbe Farbe dieser Beleuchtung adaptixt, so leidet allerdings die Sättigung der gelben Farbentöne, insbesondere des Urgelb und des grünlichen Gelb. Richtet man unter solchen Umständen ein Taschenspectroskop mit gerader Durchsicht auf eine "weisse" Fläche, so sieht man im mässig hellen Spectrum die Stelle des Gelb nahezu farblos. 
sonderem Interesse gewesen wäre, falls nämlich seine Fovea wirklich blaublind gewesen wäre; denn wir hätten dann einen Fall vor uns gehabt, in welchem ein im Allgemeinen Farbentüchtiger an begrenzter Stelle seiner Netzhaut zugleich ,blaublind“ gewesen wäre, und bätten also erfabren können, wie bei „Blaublindheit" die einzelnen Farben des Spectrums von dem geseben werden, der alle Farben aus eigener Erfahrung kennt.

$\mathrm{Da}$, wie gesagt, die von $\mathrm{K} \ddot{\mathrm{n}} \mathrm{ig}$ mitgetheilten Farbengleichungen, wenn sie richtig wären, Roth-Grünblindheit (wenn nicht gar totale Farbenblindheit) seiner Fovea beweisen wïrden, so könnte man um seine Augen besorgt sein und ein centrales Farbenskotom bei ihm vermuthen. Ich theile jedoch diese Befurehtung nicht. Denn erstens erinnere ich mich, dass er nit derselben Unbedenklichkeit, mit welcher er jetat behauptet, dass der Fovea jedes: tïchtigen ein nur "dichromatisches Farbensystem" zukomme, einmal behauptet hat, dass dem ganzen Farbensystem der Roth-Grïnblinden nicht $z$ w e i variable Reizwerthcomponenten der verschiedenen Lichter entsprecben, sondern nur eine Variable ${ }^{1}$ ), was, um mich der König'schen Terminologie zu bedienen, besagen würde, dass die "Roth-Grünverwechsler" eigentlich ein "monochromatisches Farbensystem" haben. Er wollte dies an nicht weniger als 50 "Roth-Grünverwechslern" mit Hülfe von Farbengleichungen festgestellt haben, und versuchte sogar, als er auf die Unmöglichkeit seiner Annahme aufmerksam gemacht worden war, in einer Erwiderung ${ }^{2}$ ) seinen Kritiker ins Unrecht zu setzen und ibm einen schlimmen Versuchsfehler vorzuwerfen. Später hat er freilich, ohne seine frühere Angabe zu berichtigen, von den "Rothgrïnverwechslern" unbedenklich als von "Dichromaten" in dem bekannten Sinne dieses Wortes gesprochen.

Was ferner für den normalen Zustand seiner Netzhaut spricht, sind einige Angaben, die er in der hier besprochenen Abhandlung. ïber das Farbenselen seiner Fovea macht. Noch ehe er nämlich auf die Blaublindbeit seiner Fovea zu sprechen kommt, sagt er S. 590 [14]: ,Innerhalb der Fovea tritt monochromatisches Licht, mit Ausnahme eines bestimmten Gelb (etwa $580 \mu \mu$ für mein Auge) sofort mit farbigem Character ïber die Schwelle". Weiterhin spricht

1) Centralblatt f. Augenheilkunde 1885. S. 56.

2) Ebenda. S. 260 . 
er sogar von monochromatischen "grünen", "rothen", "blauen" und "gelben" Punkten, die er innerhalb der Fovea "farbig" gesehen habe. Von monochromatischen, ,gelben" Punkten sagt er sogar ausdrücklich, dass sie, wenn man ihre Intensität allmählich herabsetzt, „vor ihrem Verschwinden in der Fovea annähernd farblos werden"; also erschienen sie ihm bei grösserer Lichtstärke farbig. Hiernach würde seine Fovea nicht roth-grünblind sein können, wenngleich seine Farbengleichungen, wären sie richtig, Roth-Grünblindheit beweisen würden.

Was König an den letzterwähnten Stellen über das farblose oder farbige Auftauchen oder Versehwinden monochromatisch leuchtender „Punkte" sagt, deren Intensität allmählich über oder unter den Schwellenwerth gebracht wird, enthält nichts Neves, wenigstens nicht für den, der weiss, dass die centrale Netzhaut einen stärkeren Farbensinn hat, als die excentrischen Theile, dass ferner das Verhältniss der weissen Sondervalenz eines spectralen Lichtes zu seiner farbigen Sondervalenz je nach der Wellenlänge ein sehr verschiedenes ist, und dass endlich in einem sonst dunklen Raume die Empfindlichkeit des Auges für die weissen Sondervalenzen der farbigen Lichter rasch wächst, was sich um so deutlicher geltend macht, je excentrischer (bis zu einer gewissen Grenze) das farbige Licht gesehen wird.

Hiermit wären, abgesehen von zwei am Schlusse zu besprechenden Punkten, die Thatsachen erschöpft, welche uns Kö n i g im III. und IV. Abschnitt seiner Abhandlang mittheilt; das Uebrige sind Hypothesen.

Da ist zuerst die Annahme, dass nur die Stäbchen im Stande seien, blane Empfindung zu vermitteln, weil nur sie Sehpurpur bezw. Sebgelb enthalten. Der Sehpurpur soll, wenn er durch Licht nur "schwach zersetzt" wird, die Empfindung "Grau" erzeugen, unter dem Einfluss stärkeren Lichts aber die Empfindung "Blau“. „Derselbe Vorgang", sagt K $\ddot{o} \mathrm{n}$ ig, "der bei geringerer Intensität als Grau empfunden wird, braucht physiologisch nicht von andrer Qualität zu sein als derjenige, welcher bei grösserer Intensität die Empfindung Blau hervorruft". Da nun die Fovea weder Stäbchen noch Sehpurpur enthält, sei sie blaublind. Die „Perception des Roth und Grün" finde im Pigmentepithel der Retina statt, wobei die Zapfen als dioptrische Apparate mitwirken, während die Perception des Blau in den Stäbchen selbst stattfinden soll. „Die 
Entstehung des Weiss“ sei „noch immer im Sinne der Young*Helmholtz'schen Theorie zu erklären"; denn „der mit der Weissempfindung verbundene physiologische Vorgang ist keine Steigerung des Vorgangs bei der Grauempfindung." Wo die Empfindung Grau aufhört und die durch einen ganz andern „physiologischen Vorgang" vermittelte Empfindung Weiss anfängt, darüber giebt uns $\mathrm{K} \ddot{o} \mathrm{n}$ ig keinen Aufschluss.

Hiernach würde die centrale Netzhaut, soweit sie keine Stäbchen enthält, auch keine weisse Empfindung vermitteln können, da ja das Blau, als die dritte, nach $\mathrm{H}$ e $\mathrm{lm}$ holt z zur Weissempfindung nöthige Empfindungscomponente hier fehlen soll. Die Empfindung „Grau" könnte von der centralen Netzhaut auch nicht vermittelt werden, da sie eben keine Stäbchen und keinen Sehpurpur enthält. Es würden für die centrale, stäbchenfreie Netzhaut nur die "Grundempfindungen" Roth und Grün, sowie die nach $\mathrm{He} 1 \mathrm{mholtz}$ ans denselben componirten Empfindungen Orange, Gelb und Gelbgrün nebst den bezüglichen Zwischentönen iubrig bleiben. Dass es genügt, weisse oder grane Papierschnitzel, klein genug, um ihr Netzhautbild auf der Forea unterzubringen, auf einen etwas dunkleren Grund zu legen und ihren Mittelpunkt zu fixiren, um sich zu überzeugen, dass man mit der Fovea jedes beliebige Weiss oder Grau zu empfinden vermag, und dass man ebenso bei guter Beleuchtung blaue, violette und purpurfarbige Schnitzel, wenn sie auf einem Grunde liegen, der ihre Farbe nicht durch Helligkeits- oder Farbencontrast zu stark beeinträchtigt, beim Fixiren auch schön blau, violett oder purpurfarbig sieht ${ }^{1}$ ): dies, wie die zahllosen analogen Erfahrungen des täglichen Lebens berücksichtigt Kö nig nicht. Er holt seine Erfahrungen aus Spectralapparaten und Farbenmischungsapparaten, und wenn es ihm hier gelingt, unter unbewusster Mithülfe von allerlei Fehlerquellen Farbengleichungen herzustellen, welche seinen theoretischen Erwartungen entsprechen, so genügt ihm das, die farbentüchtigste Stelle der Netzhaut für "blaublind" zu erklären.

1) Dem von Seite 'König's nicht unmöglichen Einwande. dass man in solchen Fällen die Schnitzel nicht eigentlich fixire, sondern nur zu fixiren glaube, während dabei die Gesichtslinie in Wirklichkeit auf einen neben dem Schnitzel liegenden Punkt treffe, ist so leicht zu begegnen, dass ich mich dabei nicht aufhalten will. 
414 Ewald Hering: Ueber angebliche Blanblindheit der Fovea centralis.

Es sind nur noch zwei Punkte kurz zu besprechen. Köni g erschloss aus seinen Annahmen, dass ein total Farbenblinder anf seiner Fovea vö $1 \mathrm{li}$ g blind sein müsse, weil dieselbe nur Zapfen enthält, und er fand dann auch bei einem total Farbenblinden, „dass bei ihm thatsächlich in dem Gesichtsfelde des rechten Auges dicht an der rechten Seite des Fixationspunktes eine selbst fuir hell leuchtende kleine weisse Flächen blinde Stelle lag." $O b$ die Farbenblindheit angeboren oder erworben war, wird nicht mitgetheilt, wohl aber, dass das linke Auge ,in Folge starker Hornhauttrübungen seit einiger Zeit für solche Versuche ganz unbrauchbar geworden ist." Derartige Untersuchungen wird Kön ig wohl fortsetzen, und wir werden deren Ergebniss abwarten müssen.

Den Schluss der Abhandlung bildet folgender Versuch: $\mathrm{K} \ddot{\mathrm{n}} \mathrm{i} \mathrm{g}$ beleuchtete eine passende Stelle seiner Sklera mit hellem rothen oder grünen Lichte und fand, dass ,an der von aussen beleuchteten Stelle niemals die Empfindung Roth bezw. Grün entstand." Dies erklärt er daraus, dass das Licht in verkehrter Richtung auf die Netzhaut gelangte, und deshalb die Wirkung der Zapfen fehlte, welche das Licht auf das, die rothe und griune Empfindung vermittelnde Pigmentepithel concentriren sollen. Es ist aber doch längst bekannt, dass diejenigen excentrischen Netzhauttheile, welche einer directen Beleuchtung durch die Sklera ïberhaupt zugänglich sind, zum mindesten relativ roth- und grünblind sind und daher eine nicht sehr ausgebreitete oder gar schwache rothe oder grüne Beleuchtung gar nicht als roth oder grïn zur Empfindung bringen. Wie also unter den beschriebenen Versuchsbedingungen von der durchleuchteten Stelle eine wahrnehmbare rothe oder, wenn man die Absorption der grünwirkenden Strahlen durch das Pigment bedenkt, gar grüne Empfindung vermittelt werden soll, wäre nicht einzusehen. Hiermit entfällt aber auch jede Beweiskraft des Versuches für die $\mathrm{K} \ddot{\mathrm{o}} \mathrm{n}$ ig'sche Doppelhypothese von der Function des Pigmentepithels und der Zapfen, mögen die letztern das Licht auf das Pigmentepithel concentriren oder nicht, und möge dieses Epithel „die noch unbekannten Sehsubstanzen für die Grundempfindungen Roth und Grün" enthalten oder nicht.

Auf die ersten Abschnitte der hier besprochenen Abhandlung $\mathrm{K} \ddot{o} \mathrm{n} \mathbf{i} g$ 's werde ich vielleicht bei andrer Gelegenheit zuriickkommen. 